\title{
Mathematical Modeling and Parameter Estimation of Battery Lifetime using a Combined Electrical Model and a Genetic Algorithm
}

\author{
M. F. B. BINELO, A. T. Z. R. SAUSEN, P. S. SAUSEN, and M. O. BINELO
}

Received on December 12, 2017 / Accepted on November 22, 2018

\begin{abstract}
In this paper, a parametrization methodology based on the Genetic Algorithm meta-heuristic is proposed for the Chen and Rincón-Mora model parameter estimation, this model is utilized for the mathematical modeling of the Lithium-ion Polymer batteries lifetime used on mobile devices. The model is also parameterized using the conventional procedure, which is based on the visual analysis of pulsed discharge curves, as presented in the literature. For both parametrization procedures, and for the model validation, experimental data obtained from a platform test are used. The simulations results show that the proposed Genetic Algorithm is able to parametrize the model with better efficacy, presenting lower mean error, and it is also a more agile process than the conventional one, been less subject to subjective aspects.
\end{abstract}

Keywords: parameter estimation, genetic algorithm meta-heuristic, mathematical modeling.

\section{INTRODUCTION}

The constant evolution of rechargeable batteries is extremely relevant for the development of more efficient mobile devices and for use of sustainable energy technologies, such as, electric and hybrid vehicles. The energy efficiency of these electrical systems is directly related to the nominal capacity of their batteries. Over the years, many advances have been made, mainly, in terms of battery capacity and energy density. An example is the Lithium-ion Polymer (LiPo) battery, which has a high energy density and power, being lighter and more compact than the Lithiumion (Li-ion) battery [22]. On the other hand, it presents functional instability when subjected to overload and full discharge conditions, in addition to having a limited number of life cycles, which results in its more frequent substitution $[21,10]$. Therefore, battery resources need to be managed efficiently and new solutions/strategies are required to improve important factors such as safety, reliability and durability.

\footnotetext{
*Corresponding author: Marcia de Fatima Brondani Binelo - E-mail: brondani.marcia@ gmail.com - https://orcid. org/0000-0001-7505-997X

**Work presented at XXXVII Congresso Nacional de Matemática Aplicada e Computacional (CNMAC).

UNIJUÍ - DCEEng/Programa de Pós-Graduação Stricto-Sensu em Modelagem Matemática , Rua Lulu Ilgenfritz, 480, 98700-000 Ijuí, RS, Brasil. E-mail: brondani.marcia@gmail.com, airam@unijui.edu.br, sausen@unijui.edu.br, manuel.binelo@unijui.edu.br
} 
In this context, many researches have investigated the prediction of the battery lifetime using mathematical models $[8,34,6,24,26,2,5,14,29]$. The battery lifetime is here understood to be the time during which the battery can supply energy to the system before a new recharge is made necessary [3]. Two main nonlinear effects present in the discharge process, the recovery and the capacity rate, have a significant influence on the battery lifetime [4]. In addition, some unavoidable phenomena can limit the battery lifetime, such as charging and discharging, aging cycles, and the thermal and power management system [21,30]. These approaches generally require reliable mathematical models that are able to accurately describe the dynamic behavior of the battery under different conditions of use. Also, for many applications, it is important to find a balance between the complexity and the model accuracy, so that it can be embedded in microprocessors and provide accurate real-time results [25, 9]. The class of electrical models has been used in a wide range of applications, considering different types of batteries $[5,11,28,19]$. These models provide important information such as current, voltage, and state of charge (SOC), and may also incorporate some nonlinear effects of the problem, maintaining a relatively low complexity [14].

The class of electrical models can be divided into six subclasses, which are: Simple models [11, 33], Thévenin-based models [17, 12], Impedance-based models [16], Runtime-based models [23], Combined models [5, 37] and Generic models [36]. Simple models use an ideal voltage source and an internal resistance to represent the battery, and they do not capture the nonlinear effects that occur during the discharge process [11], one example of Simple model is the Resistance model $[9,33]$. Thévenin subclass models provide the transient responses of the battery using Resistor-Capacitor (RC) networks [17], these models are usually able to capture two time dependent effects, the depletion and the recovery [33]. A review of different Thévenin-based models is presented in [1]. Impedance-based models are developed using the Electrochemical Impedance Spectroscopy (EIS) method, where each component of the circuits is related to an electrochemical process of the battery, and thus can provide a good description of its internal behavior [9, 16]. One of the first Impedance-based models is proposed by Randles in 1947 [1, 27]. The Runtime-based models can simulate the battery lifetime and voltage during the continuous current discharge process with reasonable accuracy, but they cannot present good results when simulating variable current discharges due to their low precision modeling of the transient battery characteristics [5, 23]. The subclass of Combined models consists of the combination of different electrical models in order to combine the best attributes of each model, such as the correct prediction of the battery lifetime, the steady-state and transient responses, and the accurate estimation of the SOC [5, 37]. One important Combined model is the Chen and Rincón-Mora model [5], which is applied in this paper for the mathematical modeling of LiPo battery lifetime used in mobile devices. This model is capable of capturing the electrical and dynamic characteristics of the battery, such as open circuit voltage (Voc), usable capacity and transient response [5]. Generic models can be used to model batteries of different technologies under different charging and discharging conditions $[3,36]$. 
An important factor in the modeling of the battery lifetime is the estimation of the its empirical parameters. Usually, the parameters of electrical models are estimated through extensive experimentation, which requires a lot of time and experience/care in obtaining the data $[20,15]$. An example is the Chen and Rincón-Mora model, which has 21 parameters usually obtained from the curve fitting method, requiring a set of data obtained from the visual analysis at specific points of battery discharge pulsed curves [5]. An alternative to overcome this problem is the use of meta-heuristic optimization techniques due to its flexibility, derivation-free mechanism and local optimal avoidance [31]. Genetic Algorithms (GAs) [13] are computational models inspired by the natural evolution mechanisms [7] applied for the solution of different classes of optimization problems. This heuristic method has shown good results due to its wide applicability in the mathematical modeling of various battery types.

In [2], a multi-objective GA is employed in the parameter estimation of an equivalent circuit model that is compared with curves of battery catalogs and with charge and discharge experiments. Different types of batteries used in electric vehicles are tested, and several test cases demonstrate that the multi-objective GA can serve as a robust and reliable tool for extracting the battery performance parameters of the electric vehicles. In [20], a GA is also employed for parameter estimation of two equivalent circuit models, one linear and other nonlinear, considering different types of industrial batteries used in electric vehicles, the results demonstrate that both linear and nonlinear equivalent circuit models can predict with accuracy the performance of various batteries of different sizes, characteristics, capacities, and materials. A similar approach is adopted in [35], where an electric circuit-based battery and a capacity fade model suitable for electric vehicles in vehicle-to-grid applications are used. The circuit parameters of the battery model are extracted using a method of GA based optimization, and the obtained battery characteristics are in close agreement with the measured characteristics (manufacturers catalog).

It is emphasized that a GA meta-heuristic depends on several characteristics specific of the problem investigated, its development needs to consider the search space, the problem optimization functional, and the selection and reproduction strategies best suited to the specific problem in question. In this context, unlike other works of literature, this paper proposes a methodology based on GA for the parameter estimation of the Chen and Rincón-Mora model, applied to the mathematical modeling of LiPo batteries lifetime used in mobile devices. The proposed GA and the mathematical model are implemented in the Matlab computational tool (student license). The validation of the methodologies used for model parametrization and Chen and Rincón-Mora model are performed by means of a comparative analysis between the simulated results and the experimental data collected on a platform test. The parameter estimation by GA methodology is compared to the conventional methodology, which uses the visual analysis of discharge curves. In this paper, the use of a single pulsed discharge curve, or multiple pulsed discharge curves, in the parameter estimation is also investigated.

The rest of the paper is organized as follows. Section 2 presents the Chen and Rincón-Mora model. Section 3 presents the experimental data used for the parameter estimation and model validation. In Section 4 the methodologies used for parameter estimation are described. Section 
5 presents the computational simulation results and discussions. In Section 6 the conclusions are drawn.

\section{CHEN AND RINCÓN-MORA MODEL}

A combined electrical model, capable of predicting battery runtime and I-V characteristics, is the Chen and Rincón-Mora model [5], which is shown in Figure 1. The Runtime-based model (circuit on the left) is composed of a self-discharge resistance $R_{a}$, a capacitor $C_{3}$ and a controlled current source $i(t)$. It models the capacity, the SOC and the battery lifetime. The Thévenin-based model (circuit on the right) models the transient response using a resistance $R_{0}$, two parallel $R C$ networks, and a controlled voltage source $\operatorname{Voc}\left(V_{S O C}\right)$. This combined model has been applied on the modeling of batteries of different technologies, presenting good accuracy [5].

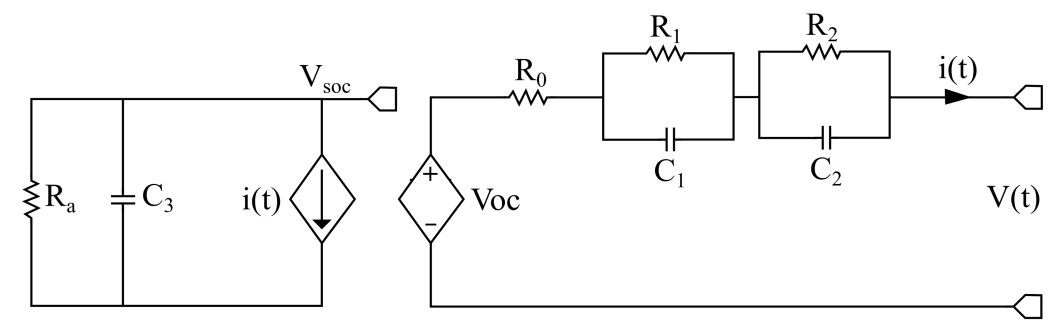

Figure 1: Chen and Rincón-Mora model equivalent circuit [1].

In order to model the transient response, two time constants, one of short and other of long duration are used, and they are defined by the $t_{0}<t<t_{r}$ time interval. During the $t_{0}<t<t_{d}$ time interval, the battery is discharged with a constant current $(i(t)>0)$. During the $t_{d}<t<t_{r}$ time interval, the battery is idle $(i(t)=0)$, in which $t_{d}$ is the end discharge time. The resistances and capacitances $R_{1}, C_{1}, R_{2}$ and $C_{2}$ represent the short and long transient responses, respectively. When in steady-state, $C_{1}$ and $C_{2}$ offer a high resistance to the continuous current, performing the open circuit role, but in transient state, this capacitors behave as a short circuit until fully charged [5].

The battery usable capacity is found from the relation among $C_{3}, R_{a}, R_{0}, R_{1}$ and $R_{2}$ components. The value of $C_{3}$ is given by

$$
C_{3}=3600 \text { Capacity } f_{1}(\text { Cycle }) f_{2}(\text { Temp }),
$$

where: Capacity is the nominal capacity, $f_{1}($ Cycle $)$ is the number of cycles correction factor, $f_{2}($ Temp $)$ is the temperature correction factor [5].

The variation of the usable capacity is caused by the intensity of the discharge current applied to the battery, being neglected other aspects that could affect it, such as the number of cycles, temperature and storage time. Therefore, the value of $R_{a}$ is not taken into consideration, and both correction factors are equal to 1 . Different currents applied to $C_{3}$ result in voltage drops in the 
equivalent resistor, which corresponds to the sum of resistances $R_{0}, R_{1}$ e $R_{2}$. This results in a set of different SOC values, according to the voltage drop, gradually reducing the usable capacity of the battery during its discharge [5]. The output voltage $V_{s}(t)$ is then expressed as

$$
V_{s}(t)=\operatorname{Voc}\left(V_{S O C}\right)-R_{0} i(t)-V_{\text {Transient }}(t)
$$

where: $V_{\text {Transient }}(t)$ is the transient voltage calculated from

$$
V_{\text {Transient }}(t)=V_{1}+V_{2},
$$

where: $V_{1}$ is the short duration transient voltage, and $V_{2}$ is the long duration transient voltage, both given by the equations (2.4) and (2.5) respectively,

$$
\begin{aligned}
& V_{1}= \begin{cases}R_{1} i(t)\left[1-e^{-\frac{\left(t-t_{0}\right)}{\tau_{1}}}\right], & t_{0}<t<t_{d} \\
V_{1}\left(t_{d}\right) e^{-\frac{\left(t-t_{d}\right)}{\tau_{1}}}, & t_{d}<t<t_{r}\end{cases} \\
& V_{2}= \begin{cases}R_{2} i(t)\left[1-e^{-\frac{\left(t-t_{0}\right)}{\tau_{2}}}\right], & t_{0}<t<t_{d} \\
V_{2}\left(t_{d}\right) e^{-\frac{\left(t-t_{d}\right)}{\tau_{2}}}, & t_{d}<t<t_{r}\end{cases}
\end{aligned}
$$

where: $V_{1}\left(t_{d}\right)$ is the short duration transient voltage at the final discharge time, $\tau_{1}=R_{1} C_{1}, V_{2}\left(t_{d}\right)$ is the long duration transient voltage at the final discharge time, and $\tau_{2}=R_{2} C_{2}$ [15].

The elements $V o c$ and $R_{0}$ from equation (2.2), and also $R_{1}, C_{1}, R_{2}$ and $C_{2}$, that model the transient voltage response, are functions of $S O C$, as described by the following equations

$$
\begin{gathered}
\operatorname{Voc}(S O C)=a_{0} e^{-a_{1} S O C}+a_{2}+a_{3} S O C-a_{4} S O C^{2}+a_{5} S O C^{3}, \\
R_{0}(S O C)=b_{0} e^{-b_{1} S O C}+b_{2}, \\
\left\{\begin{array}{l}
R_{1}(S O C)=c_{0} e^{-c_{1} S O C}+c_{2}, \\
C_{1}(S O C)=d_{0} e^{-d_{1} S O C}+d_{2}, \\
R_{2}(S O C)=e_{0} e^{-e_{1} S O C}+e_{2}, \\
C_{2}(S O C)=f_{0} e^{-f_{1} S O C}+f_{2} .
\end{array}\right.
\end{gathered}
$$

It is observed that the methodology usually adopted in the literature for the estimation of the 21 empirical parameters of equations (2.6), (2.7) and (2.8) is based on curve fitting techniques using 4 pulsed battery discharge curves as described in [5].

\section{EXPERIMENTAL DATA}

For Chen and Rincón-Mora model parametrization and validation, experimental data are used. They refer to new LiPo batteries, model PL383562 - 2C, used in mobile devices, collected from a testbed developed by the Industrial Automation and Control Group (GAIC), of the Regional 
University of Northwest of Rio Grande do Sul State (Unijuí). The experimental data collection is a rigorous procedure, so a single standard is adopted in all experimental tests. Initially, the batteries are connected to an external power source and are subject to a full charge process with an applied current corresponding to $20 \%$ of the rated battery capacity [18], which is equivalent to $160 \mathrm{~mA}$. The charge process is considered complete when battery voltage reaches $4.2 \mathrm{~V}$. For the discharge process, the batteries are disconnected from the charging station and connected to the testbed [3].

The experimental tests performed to estimate the model empirical parameters are based on the procedure adopted by [5], which considers that each discharge curve contains from 6 to 10 pulses. Thus, following the methodology presented in [5], 4 pulsed discharge curves are used, whose discharge current in $m A$, pulse periods and pulse duration both in min, are presented in the Table 1. The periods are repeated until the batteries reach the cutoff voltage corresponding to $3 V$. The parametrization performed in this paper uses first the visual analysis process of the pulsed curves according to [5], and later using GA.

Table 1: Pulsed discharge curves used for parameter estimation of the model.

\begin{tabular}{ccc}
\hline Discharge current & Pulse period & Pulse duration \\
\hline 80 & 68.34 & 58.34 \\
160 & 40.00 & 30.00 \\
320 & 25.00 & 15.00 \\
640 & 17.50 & 7.50 \\
\hline
\end{tabular}

The parametrization process is also analysed when only one discharge curve is used. For this purpose, a pulsed discharge intermediate current curve of $400 \mathrm{~mA}$, with pulse period of $21.67 \mathrm{~min}$ is selected, in which the first $11.67 \mathrm{~min}$ is applied to the discharge current (pulse duration), and in the next $10 \mathrm{~min}$ the battery remains idle. This sequence is repeated until the battery reaches the voltage of $3 \mathrm{~V}$. The model validation considers a constant discharge profiles set, which contains low, medium and high currents, according the capacity of $800 \mathrm{~mA}$. Thus, 31 profiles are defined in the range from $50 \mathrm{~mA}$ to $800 \mathrm{~mA}$, equally divided into intervals of $25 \mathrm{~mA}$. For each profiles 8 experiments are performed, obtaining the average experimental lifetime $\left(L_{e a}\right)$ that is used as metric for calculating the model error.

A statistical analysis of the experimental data using One-Way ANOVA and Tukey test is performed, and 11 statistically different profiles are selected from 31 profiles measured. Table 2 shows 11 statistically different profiles chosen, in which $L_{e i}$ represents battery lifetime measured for each sample, in $\min$ with $1 \leq i \leq 8$, and $L_{e a}$ represents the average experimental lifetime, in min. Next section, the two methodologies adopted for the estimation of Chen and Rincón-Mora model empirical parameters are described. 
Table 2: Experimental data used for the Chen and Rincón-Mora model validation.

\begin{tabular}{cccccccccc}
\hline Profiles & $L_{e 1}$ & $L_{e 2}$ & $L_{e 3}$ & $L_{e 4}$ & $L_{e 5}$ & $L_{e 6}$ & $L_{e 7}$ & $L_{e 8}$ & $L_{e a}$ \\
\hline 50 & 964.07 & 980.42 & 860.43 & 922.15 & 920.43 & 993.96 & 931.38 & 950.08 & 940.36 \\
75 & 593.06 & 607.28 & 626.98 & 599.75 & 586.9 & 652.15 & 582.58 & 606.78 & 606.93 \\
100 & 464.71 & 474.42 & 460.45 & 466.3 & 470.75 & 459.03 & 445.53 & 486.62 & 465.97 \\
125 & 388.48 & 392.93 & 375.03 & 367.4 & 380.9 & 393.65 & 381.55 & 398.17 & 384.76 \\
150 & 283.02 & 311.72 & 302.97 & 307.58 & 345.03 & 297.62 & 279.48 & 305.38 & 304.1 \\
175 & 280.85 & 274.5 & 271.33 & 285.07 & 297.07 & 253.5 & 246.43 & 269.1 & 272.23 \\
200 & 232.2 & 219.18 & 213.95 & 236.18 & 218.83 & 235.7 & 239.72 & 228.12 & 227.98 \\
250 & 191.23 & 173.47 & 167.72 & 188.93 & 182.78 & 190.15 & 193.12 & 184.65 & 184.01 \\
325 & 141.57 & 145.68 & 149.53 & 143.47 & 148.45 & 129.85 & 126.4 & 145.33 & 141.28 \\
400 & 115.55 & 117.75 & 121.52 & 115.78 & 118.8 & 107.9 & 101.9 & 117.5 & 114.58 \\
525 & 88.57 & 91.3 & 93.15 & 85.28 & 92.63 & 78.67 & 74.11 & 85.85 & 86.19 \\
\hline
\end{tabular}

\section{METHODOLOGIES FOR PARAMETER ESTIMATION}

This section presents the parameter estimation of the Chen and Rincón-Mora model carried out from two different methodologies. The Methodology 1 is based on the curve fitting technique adopted by [5], and the Methodology 2 corresponds to the proposed GA methodology. Both methodologies determine the laws of functions $V o c, R_{0}, R_{1}, C_{1}, R_{2}$ and $C_{2}$ presented in equations(2.6)-(2.8), which contains the 21 parameters that need to be estimated.

\subsection{Methodology 1: Curve Fitting}

This methodology is based on the curve fitting technique and follows the procedure described in [5]. For each one of the functions mentioned above, four experimental curves are generated, corresponding to 4 profiles of pulsed discharge currents of $80 \mathrm{~mA}, 160 \mathrm{~mA}, 320 \mathrm{~mA}$ and $640 \mathrm{~mA}$. Each curve contains a characteristic points set that is obtained from the discharge current pulses. In this context, the curve fitting consists in finding a law of the function that passes through all these points, and therefore, the respective parameters.

Thus, to determine the function Voc in equation (2.6), it is necessary to measure the open circuit voltage at different points in the SOC, which correspond to the steady state open-circuit voltage at each pulse of discharge [5]. The function $R_{0}$, in equation (2.7), is determined from the $R_{0}$ resistance calculation in each pulse of the discharge currents. This resistance is responsible for the instantaneous voltage drop in the pulse, considering the initial and final voltages at the instant the discharge current is applied, as shown in [32]. In order to obtain the laws of functions $R_{1}$ and $C_{1}$, in equation (2.8), the values $R_{1}$ and $C_{1}$ are calculated considering the first seconds of each pulse of the discharge currents. As described in [32], the voltage difference in the corresponding interval is required for the calculation of $R_{1}$, and for the calculation of $C_{1}$, the time difference is needed, which is obtained tracing a line tangent to the point corresponding to the initial voltage of the interval. Finally, the functions $R_{2}$ and $C_{2}$, in equation (2.8), depend on the values $R_{2}$ and $C_{2}$, 
which are calculated considering the remaining seconds of each pulse of the discharge currents, similarly to the short duration constants [32].

All measurements are made from the visual analysis at the specific points of the discharge currents pulses applied to the batteries. After that, the Nonlinear Least Squares method implemented in Matlab software is used to curve fitting, thus obtaining the equation that represents each function of the Chen and Rincón-Mora model with the respective empirical parameters.

\subsection{Methodology 2: Genetic Algorithm}

By an analogy between the natural selection process and the problem of model parametrization, it is possible to consider the interaction between the experimental data and the results simulated by the model as being the environment. The genetic code of each individual corresponds to the 21 model parameters that need to be estimated, presented in the equations (2.6)-(2.8). Each parameter is a chromosome, initially with genotypical representation discretized in four decimal digits, which are the genes. The aptitude of each parameter is directly related to the objective function $F$, which is the maximum mean voltage difference between the model curve and the experimental data when a set of different discharge curves are jointly considered,

$$
F=\max _{i} \frac{\int_{0}^{L_{e}}\left|V_{s i}-V_{e i}\right| d t}{L_{e}},
$$

where: $V_{s i}$ is the voltage simulated by the model for the discharge curve $i$, and $V_{e i}$ is the experimental voltage for the discharge curve $i$, so that a set of different discharge currents can be used in conjunction for the parametrization problem. The parametrization problem can be expressed as the minimization problem of the functional given by

$$
\underset{a_{0.5}, b_{0.2}, c_{0.2}, d_{0.2}, e_{0.2}, f_{0 . .2}}{\arg \min } F\left(a_{0 . .5}, b_{0 . .2}, c_{0 . .2}, d_{0 . .2}, e_{0 . .2}, f_{0.2}\right)
$$

where: $a_{0 . .5}, b_{0 . .2}, c_{0 . .2}, d_{0 . .2}, e_{0 . .2}, f_{0 . .2}$ correspond to the 21 parameters that minimize the error between the model and the experimental data.

The fitness function is based on the Power Scale mapping method, being obtained from the inverse of $F$ (equation (4.1)), that is, $F^{-1}$. This means that the lower the value of $F$ is, the greater is the aptitude of the parameters for the case analyzed. Thus, the GA provides the values of the 21 parameters of the Chen and Rincón-Mora model optimized, when reaching the stopping criterion. The configuration of the GA is done empirically by performing several tests, considering fundamental aspects such as stability, convergence, responses quality and limits imposed by the computational cost of the algorithm.

In this way, the GA is implemented in Matlab software and initialized with a random population. The genetic code of the individual is composed of 84 decimal digits, using 4 digits to represent each of the 21 model parameters. Therefore, there are ten thousand different possible combinations, ranging from 0000 to 9999 for each parameter. All manipulation operations of the genetic code in the algorithm (i.e., crossing and mutation), are made considering this genotypic representation. In order to calculate the fitness, it is necessary to transform the genotypic representation 
into its corresponding phenotypic form, that is, the parameter value to be used in the battery simulation model. Table 3 presents the minimum and maximum values of each parameter defined by the authors and based on Metodology 1 . The phenotypic form of the parameter is obtained from the genotype according to equation

$$
P=v_{\min }+\left(\frac{C}{9999}\right)\left(v_{\max }-v_{\min }\right)
$$

where: $P$ is the parameter value (phenotype), $C$ is the genetic code (genotype), and $v_{\min }$ and $v_{\max }$ are the minimum and maximum values of the parameter according to Table 3.

Table 3: Parameters minimum and maximum ranges.

\begin{tabular}{ccc}
\hline Parameter & $v_{\min }$ & $v_{\max }$ \\
\hline$a_{0}$ & -1.4094 & -0.8456 \\
$a_{1}$ & 3.0706 & 23.0706 \\
$a_{2}$ & 2.9696 & 4.9493 \\
$a_{3}$ & -1.3849 & -0.8309 \\
$a_{4}$ & -2.5334 & -1.5200 \\
$a_{5}$ & -0.8185 & -0.4911 \\
$b_{0}$ & 2.3018 & 3.8364 \\
$b_{1}$ & 48.1261 & 80.2101 \\
$b_{2}$ & 0.2375 & 0.3959 \\
$c_{0}$ & 13.6187 & 22.6978 \\
$c_{1}$ & 113.3475 & 188.9125 \\
$c_{2}$ & 0.0406 & 0.1006 \\
$d_{0}$ & -667.7264 & -400.6358 \\
$d_{1}$ & 4.3313 & 14.3313 \\
$d_{2}$ & 381.0251 & 635.0419 \\
$e_{0}$ & 0.9902 & 1.9902 \\
$e_{1}$ & 9.3493 & 49.3493 \\
$e_{2}$ & 0.0671 & 0.1271 \\
$f_{0}$ & -1818.3673 & -1091.0204 \\
$f_{1}$ & 3.5250 & 13.5250 \\
$f_{2}$ & 980.6167 & 1634.3611 \\
\hline & &
\end{tabular}

Multiple points are used for the crossover procedure, according to the number of model parameters. They are represented in the genetic code, so that the individual generated could inherit parameters from the mother or the father, each parameter is passed in its integral form, being perturbed only by mutation. The GA uses non-uniform mutation rates, according to the position of the digit in the parameter representation, ranging from $0.5 \%$ to $2 \%$, with the least significant digit having the highest mutation rate. 
At each iteration of the GA, the values of the optimized parameters are informed to the model and it is executed, providing the simulated curves that describe the voltage decay as a function of time until the cutoff level is reached. First, a set of four pulsed discharge curves corresponding to $80 \mathrm{~mA}, 160 \mathrm{~mA}, 320 \mathrm{~mA}$, and $640 \mathrm{~mA}$ is used, according to the procedure adopted in [5]. After, a new analysis is done using only a pulsed discharge curve of $400 \mathrm{~mA}$. Afterwards obtaining the parameters, the estimation of the model empirical parameters and the Chen and Rincón-Mora model are validated. This procedure is performed in order to validate the methodology proposed for the parameter estimation, as well as to verify the model accuracy to simulate the lifetime of LiPo batteries.

Considering the probabilistic nature of the GA, the whole procedure is repeated 10 times in order to analyse the mean results. The simulations results for the standard deviations also are found, and as will be presented in the next section, they are low, therefore, more independent realizations are not deemed necessary. The algorithm uses a population size of 100 individuals with a $10 \%$ selection rate, and for each independent realization, 100 generations are computed. The population size and number of generations are severely limited by the computational cost of the fitness function, but the configuration used is sufficient to consistently obtain good results. Given the relatively small population and number of generations, the truncation selection method is employed, in order to obtain a faster convergence. As the results will show, local maxima stagnation did not affect the algorithm performance.

\section{RESULTS AND DISCUSSIONS}

In this section, the simulations results of the Chen and Rincón-Mora model are presented, according to the two methodologies adopted in this paper. Initially, the validation of the parameter estimation and the parameters values for each methodology are presented, followed by the model validation, considering 11 discharge profiles of constant current. The results analysis takes into consideration the difference between the simulated and experimental lifetimes, and these differences are presented as relative error percentages. Ten independent realizations of the GA are performed for the single curve parametrization, and for the four curves parametrization cases. The mean errors and standard deviations are presented, considering all GA realizations for the parametrization validation and the model validation. In the results also is presented the best parameters set for each parametrization case, which is the set of parameters from the independent GA realization that presents the lowest model validation error.

\subsection{Validation of parameter estimation}

For the validation of the parameter estimation, the discharge profiles used for the parameter estimation are applied to the Chen and Rincón-Mora model, and the simulations results are compared. Initially, the simulations for Methodology 1 , which is based on the visual analysis of the pulsed discharge curves of $80 \mathrm{~mA}, 160 \mathrm{~mA}, 320 \mathrm{~mA}$ and $640 \mathrm{~mA}$, are performed. Following, the validation of parameter estimation for Methodology 2 is performed, which is based on the pro- 
posed GA, using the same pulsed discharge profiles used in Methodology 1, and it is referred here as Methodology 2 (4 curves). The experimental lifetimes $\left(L_{e}\right)$ for each profile, and the simulated lifetimes $\left(L_{s}\right)$ for Methodology 1 with respective errors are presented in Table 4; and the simulated lifetimes with respective errors for the Methodology 2 (4 curves), considering the best parameter set $\left(L_{s}\right)$, and the average results for 10 independent GA realizations $\left(L_{s a}\right)$, are presented in Table 5.

Table 4: Validation of parameter estimation from Methodology 1.

\begin{tabular}{cccc}
\hline \multirow{2}{*}{ Profiles $(m A)$} & \multirow{2}{*}{$L_{e}(\mathrm{~min})$} & \multicolumn{2}{c}{ Methodology 1 } \\
\cline { 3 - 4 } & & $L_{s}(\mathrm{~min})$ & Error $(\%)$ \\
\hline 80 & 655.50 & 672.67 & 2.62 \\
160 & 379.60 & 379.00 & 0.16 \\
320 & 227.84 & 232.67 & 2.12 \\
640 & 160.50 & 160.17 & 0.20 \\
Mean error & & & 1.28 \\
\hline
\end{tabular}

Table 5: Validation of parameter estimation from Methodology 2 (4 curves).

\begin{tabular}{cccccc}
\hline \multirow{2}{*}{ Profiles $(m A)$} & \multirow{2}{*}{$L_{e}(\mathrm{~min})$} & \multicolumn{4}{c}{ Methodology $2(4$ curves $)$} \\
\cline { 3 - 6 } & & $L_{s}(\mathrm{~min})$ & Error $(\%)$ & $L_{s a}(\mathrm{~min})$ & Error (\%) \\
\hline 80 & 655.50 & 672.20 & 2.55 & 671.25 & 2.40 \\
160 & 379.60 & 378.34 & 0.33 & 378.71 & 0.39 \\
320 & 227.84 & 232.62 & 2.10 & 232.73 & 2.14 \\
640 & 160.50 & 160.47 & 0.02 & 160.26 & 0.27 \\
Mean error & & & 1.25 & & 1.30 \\
\hline
\end{tabular}

According to the simulations results presented for the validation of the parameter estimation, the model obtained a mean error of $1.28 \%$ for Methodology 1 , and of $1.25 \%$ for Methodology 2 (4 curves) for the best parameter set, and an average error of $1.30 \%$ Methodology 2 (4 curves) for 10 independent GA realizations, with a standard deviation of $0.10 \%$. The obtained errors are considerably low for the class of electrical models. The simulation curves of the model considering both methodologies, and the experimental data curves for each of the discharge profiles used in the parametrization procedure, can be seen in Figures 2, 3, 4 and 5, where the simulation curves for the GA method are generated based on the best parameter set. The simulation curves show a behavior close to the experimental data during most of the discharge interval, with a slight difference for the $320 \mathrm{~mA}$ profile (Figure 4), and at the end of the discharge time for the $80 \mathrm{~mA}$ (Figure 2) and $320 \mathrm{~mA}$ (Figure 4) profiles, where the simulation curves take more time to reach cutoff level. 


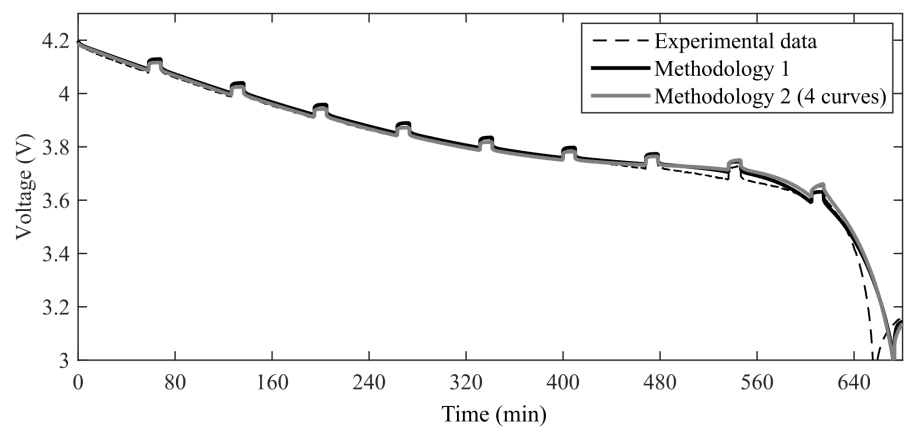

Figure 2: Comparison between experimental data and simulations for Methodology 1 and Methodology 2 (4 curves), for $80 \mathrm{~mA}$ discharge profile.

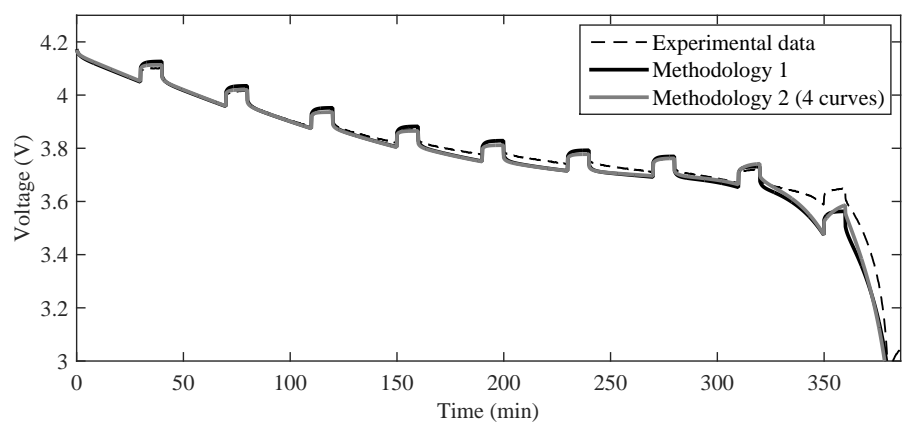

Figure 3: Comparison between experimental data and simulations for Methodology 1 and Methodology 2 (4 curves), for $160 \mathrm{~mA}$ discharge profile.

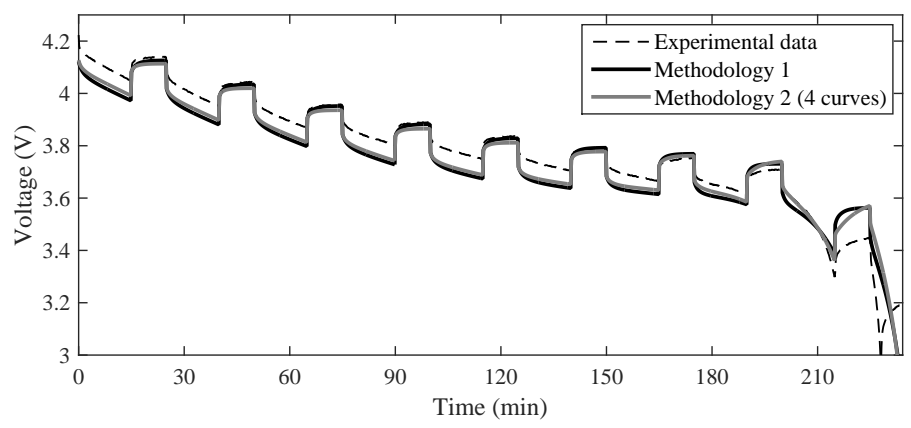

Figure 4: Comparison between experimental data and simulations for Methodology 1 and Methodology 2 (4 curves), for $320 \mathrm{~mA}$ discharge profile. 


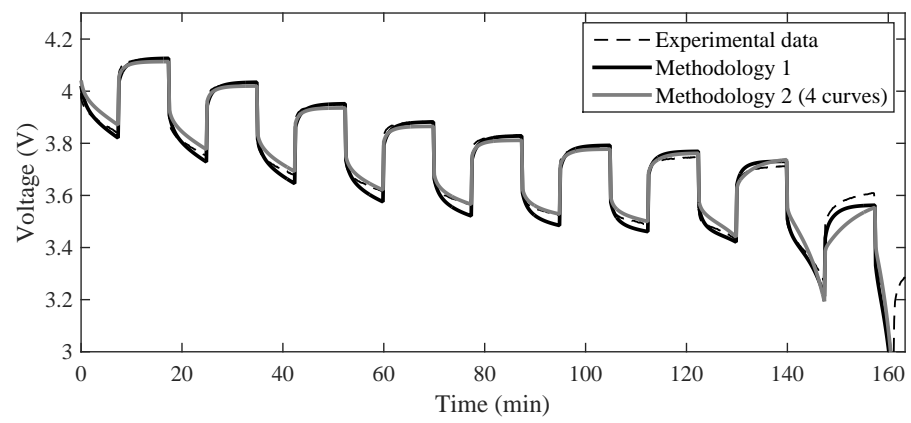

Figure 5: Comparison between experimental data and simulations for Methodology 1 and Methodology 2 (4 curves), for $640 \mathrm{~mA}$ discharge profile.

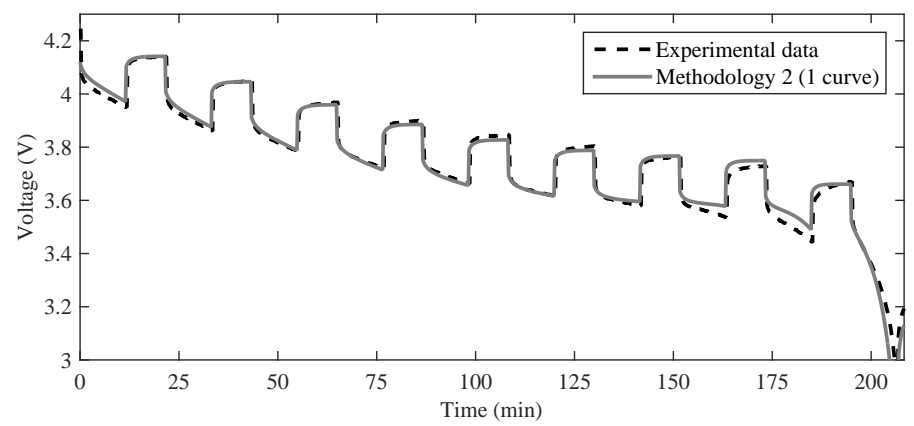

Figure 6: Comparison between experimental data and Methodology 2 (1 curve), for $400 m A$ discharge profile.

The Methodology 2 is also used applying just one pulsed discharge curve, whose periods are shown in Section 3, and is referred as Methodology 2 ( 1 curve). In Figure 6, the pulsed $400 \mathrm{~mA}$ discharge curve and the model simulation curve are presented, also considering the best parameter set from 10 independent GA realizations. It is possible to see that the simulation curve behavior is close to the experimental data, and presents a very low error for the discharge time, of only $0.85 \%$. When all 10 independent GA realizations are considered, the average parametrization error is $0.59 \%$, with a standard deviation of $0.12 \%$. It is important to note that the best parameters set is chosen according to the model validation error, presented in the next subsection, and not the parametrization error, presented here. This is the reason why the best parameter set is not necessarily the one that presents the best fitting for one specific parametrization curve, what highlights the importance of using several curves for the parametrization procedure, capturing a broader spectrum of the battery behavior. This low error for the parametrization procedure is expected, since only one curve must be taken into account by the GA optimization procedure, but nevertheless, this result shows the efficacy of the proposed GA. 
The results demonstrate that both methodologies can be reliably applied for the Chen and Rincón-Mora model parametrization, and the parameters values are shown in Table 6. The next subsection presents the model validation according to each parametrization methodology. For Methodology 2, the best parameters sets from 10 independent realizations are shown.

Table 6: Parameters estimated using Methodology 1 and Methodology 2.

\begin{tabular}{crrr}
\hline Parameter & Methodology 1 & Methodology 2 (4 curves) & Methodology 2 (1 curve) \\
\hline$a_{0}$ & -1.1275 & -1.1473 & -1.1359 \\
$a_{1}$ & 13.0706 & 15.4206 & 16.9806 \\
$a_{2}$ & 3.9594 & 3.9443 & 3.9582 \\
$a_{3}$ & -1.1079 & -1.1236 & -1.1021 \\
$a_{4}$ & -2.0267 & -2.0438 & -2.0498 \\
$a_{5}$ & -0.6548 & -0.6514 & -0.6748 \\
$b_{0}$ & 3.0691 & 3.0747 & 3.0753 \\
$b_{1}$ & 64.1681 & 59.6201 & 65.9941 \\
$b_{2}$ & 0.3167 & 0.2685 & 0.3172 \\
$c_{0}$ & 18.1582 & 18.1493 & 18.1331 \\
$c_{1}$ & 151.13 & 143.2540 & 146.73 \\
$c_{2}$ & 0.0706 & 0.0436 & 0.0750 \\
$d_{0}$ & -534.1811 & -543.3651 & -518.9451 \\
$d_{1}$ & 9.3313 & 6.5143 & 5.9483 \\
$d_{2}$ & 508.0335 & 494.3895 & 514.2895 \\
$e_{0}$ & 1.4902 & 1.7458 & 1.2526 \\
$e_{1}$ & 29.3493 & 13.5013 & 9.8573 \\
$e_{2}$ & 0.0971 & 0.0722 & 0.0744 \\
$f_{0}$ & -1454.6938 & -1425.2 & -1452.6 \\
$f_{1}$ & 8.5250 & 9.4160 & 13.375 \\
$f_{2}$ & 1307.4889 & 1365.3 & 1399.9 \\
\hline & & &
\end{tabular}

\subsection{Model validation}

For the validation procedure, the model is subjected to 11 different discharge profiles of constant currents, which are not used during the parametrization process. The simulations are performed using the parameters obtained by Methodology 1, Methodology 2 (4 curves) and Methodology 2 (1 curve). The errors corresponding to each discharge profile and parametrization methodology are presented in Figure 7. For Methodology 2, the parameters obtained from the 10 independent GA realizations are used, and the mean results of the battery lifetime errors are computed. The Methodology 2 (4 curves) and ( 1 curve) - best parameter set error presents the errors corresponding to each of the 11 discharge profile for the parameters set of the 10 independent GA realizations that obtained the lowest mean error. Methodology 2 ( 4 curves) and ( 1 curve) - mean error presents the mean error for each of the 11 validation profiles considering 10 independent GA realization. It should be noted that the results for the best parameter set error consider the 
average error of all 11 validation profiles, so it is possible that some specific profiles present an error greater than the average of the 10 independent GA realizations, such as in the profiles of $125 \mathrm{~mA}$ and $175 \mathrm{~mA}$.

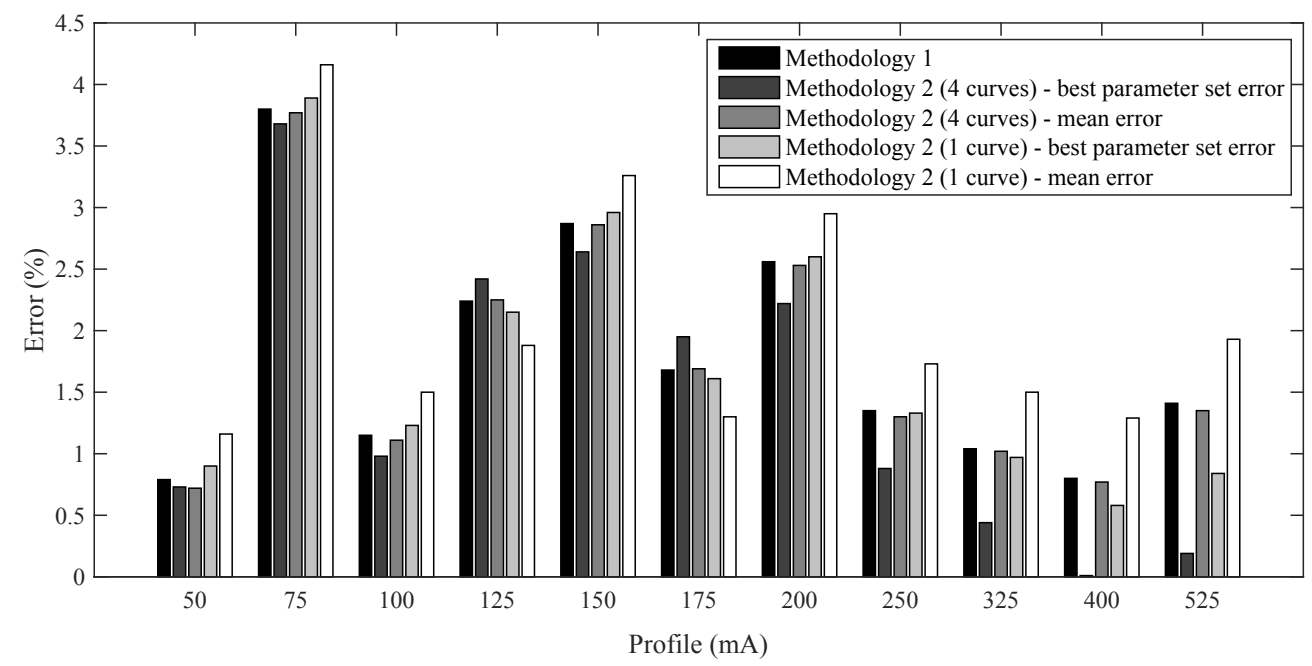

Figure 7: Error percentages for different discharge currents and different parametrization methodologies.

The overall results considering all discharge profiles are the following. For Methodology 1, the resulting mean error is of $1.79 \%$. For Methodology 2 (4 curves), the best parameters set presents the mean error of $1.47 \%$, and considering the 10 independent GA realizations, its average error is of $1.76 \%$, with the standard deviation of $0.25 \%$. For Methodology 2 ( 1 curve), the best parameters set presents the mean error of $1.73 \%$, and considering the 10 independent GA realizations, its average error is of $2.06 \%$, with the standard deviation of $0.13 \%$. Even though the Methodo$\operatorname{logy} 2$ ( 1 curve) presented the lowest error during the parametrization procedure, it presented the highest error for the validation of the model, because it failed to capture the model behaviour for different currents of discharge. The best results are obtained by Methodology 2 ( 4 curves).

In addition to more precise results, it is important to note that the methodology using the proposed GA is much less subjective and also much more agile. For the results presented in this paper, 12 hours are used for the parametrization using the visual curve analysis methodology, while the proposed GA obtained good results in 20 generations, using about 15 minutes of processing time in a conventional laptop with an I5 Intel processor and 4GB of main memory. Using 4 different curves for parametrization, and 100 generations, the processing time for each GA realization is 5 hours, as presented in Figure 8. 


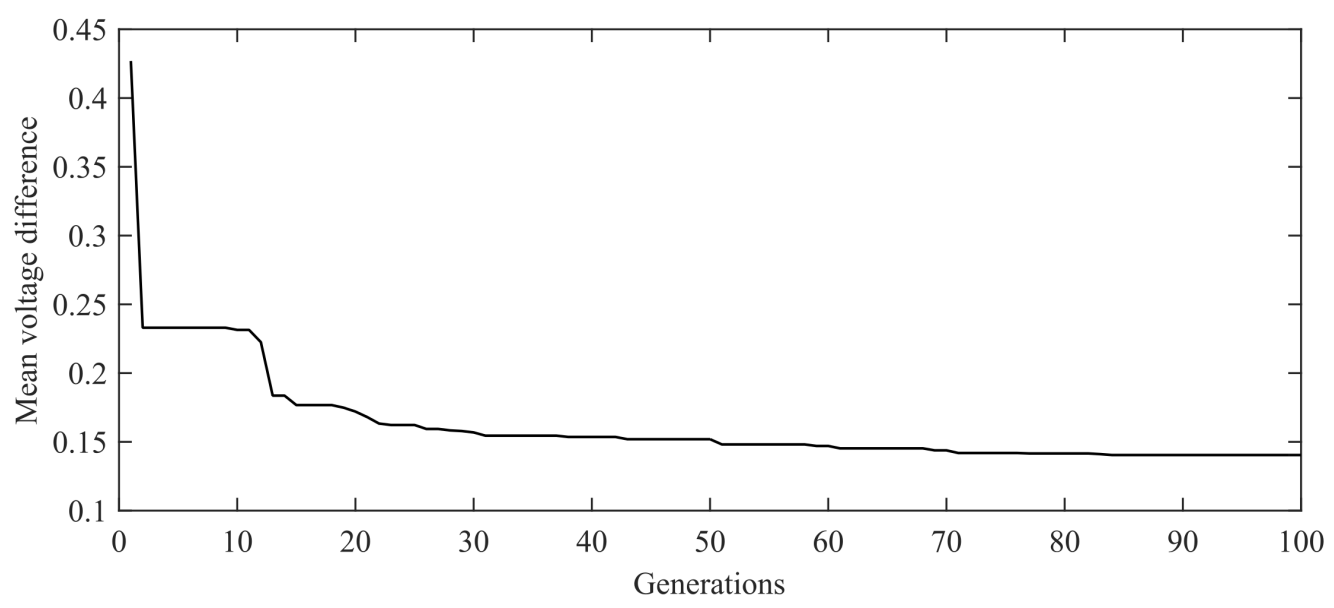

Figure 8: Mean voltage difference during 100 GA generations.

\section{CONCLUSION}

In this paper, the mathematical modeling of the lifetime of LiPo batteries is presented, using the Chen and Rincón-Mora model. The parametrization of the model is further investigated, and a parametrization procedure based on GA is proposed. The proposed GA is compared to the conventional parametrization procedure which is based on the visual analysis of pulsed discharge curves. As is the case for the conventional procedure, 4 different discharge curves are jointly taken into account by the GA optimization method. The results show that the GA based method can achieve better results than the conventional parametrization procedure, as demonstrated by the model validation, that used 11 different discharge profiles to analyse the efficacy of the parametrization methods. The results also show that using more than one discharge curve of the experimental data during the parametrization process, covering different current profiles, greatly improves the model accuracy.

\section{ACKNOWLEDGEMENTS}

This study was financed in part by the Coordenação de Aperfeiçamento de Pessoal de Nível Superior - Brasil (CAPES) - Finance Code 001, Brazil.

RESUMO. Neste artigo é proposta uma metodologia baseada na meta-heurística Algoritmo Genético para estimar os parâmetros no modelo Chen e Rincón-Mora, sendo este aplicado na modelagem matemática do tempo de vida de baterias de Lítio-íon Polímero. O modelo também é parametrizado a partir da metodologia convencional, que necessita de dados obtidos por meio da análise visual de curvas de descargas pulsadas, conforme apresentada na literatura. Para ambos os processos, parametrização e validação do modelo, são utilizados dados experimentais obtidos em uma plataforma de testes. Os resultados mostram que o Algoritmo Genético proposto parametriza de forma mais eficaz o modelo, apresen- 
tando erro médio menor, além de ser um processo mais rápido e menos subjetivo que o procedimento convencional.

Palavras-chave: estimação de parâmetros, meta-heurística algoritmo genético, modelagem matemática.

\section{REFERENCES}

[1] M.F.B. Binelo, L.B. Motyczka, A.T.Z.R. Sausen, P.S. Sausen \& M.O. Binelo. "Battery Charge and Discharge Behavior Prediction Using Electrical Mathematical Models". Advances in Mathematics Research. Nova Science Publishers Incorporated (2017), 127-154 pp.

[2] J. Brand, Z. Zhang \& R.K. Agarwal. Extraction of battery parameters of the equivalent circuit model using a multi-objective genetic algorithm. Journal of Power Sources, 247 (2014), 729 - 737.

[3] M.F. Brondani, A. Sausen, P.S. Sausen \& M.O. Binelo. Battery Model Parameters Estimation Using Simulated Annealing. TEMA - Tendências em Matemática Aplicada e Computacional, 18 (2017), $127-135$.

[4] M.F. Brondani, A.T.Z.R. Sausen, P.S. Sausen \& M.O. Binelo. Parameter estimation of lithium ion polymer battery mathematical model using genetic algorithm. Computational and Applied Mathematics, (2017), 1-18.

[5] M. Chen \& G. Rincón-Mora. Accurate electrical battery model capable of predicting runtime and I-V performance. IEEE Transactions on Energy Conversion, 21(2) (2006), 504-511.

[6] C. Chiasserini \& R. Rao. Pulsed battery discharge in communication devices. Proceedings of the 5th International Conference on Mobile Computing and Networking, (1999), 88-95.

[7] C. Darwin. "On the origin of species by means of natural selection; or, The preservation of favoured races in the struggle for life". D. Appleton and Company (1861).

[8] M. Doyle, T.F. Fuller \& J.S. Newman. Modeling of galvanostatic charge and discharge of the lithium/polymer/insertion cell. Journal of The Electrochemical Society, 140 (1993), 1526-1533.

[9] A. Fotouhi, D.J. Auger, K. Propp, S. Longo \& M. Wild. A review on electric vehicle battery modelling: From Lithium-ion toward Lithium-Sulphur. Renewable and Sustainable Energy Reviews, 56 (2016), $1008-1021$.

[10] G.P. Hammond \& T. Hazeldine. Indicative energy technology assessment of advanced rechargeable batteries. Applied Energy, 138 (2015), 559 - 571.

[11] H. He, R. Xiong, H. Guo \& S. Li. Comparison study on the battery models used for the energy management of batteries in electric vehicles. Energy Conversion and Management, 64 (2012), 113 121. \{IREC $\}$ 2011, The International Renewable Energy Congress.

[12] H. He, R. Xiong, X. Zhang, F. Sun \& J. Fan. State-of-Charge Estimation of the Lithium-Ion Battery Using an Adaptive Extended Kalman Filter Based on an Improved Thevenin Model. IEEE Transactions on Vehicular Technology, 60(4) (2011), 1461-1469. doi:10.1109/TVT.2011.2132812. 
[13] J.H. Holland. "Adaptation in Natural and Artificial Systems". University of Michigan Press, Ann Arbor, MI, USA (1975).

[14] T. Hu \& H. Jung. Simple algorithms for determining parameters of circuit models for charging/discharging batteries. Journal of Power Sources, 233 (2013), 14 - 22.

[15] T. Kim \& W. Qiao. A hybrid battery model capable of capturing dynamic circuit characteristics an nonlinear capacity effects. IEEE Wireless Communications and Networking Conference, 26 (2011), $1172-1180$.

[16] S.E. Li, B. Wang, H. Peng \& X. Hu. An electrochemistry-based impedance model for lithium-ion batteries. Journal of Power Sources, 258 (2014), 9 - 18.

[17] C. Lin, H. Mu, R. Xiong \& W. Shen. A novel multi-model probability battery state of charge estimation approach for electric vehicles using H-infinity algorithm. Applied Energy, 166 (2016), 76 - 83.

[18] D. Linden \& T.B. Reddy. "Handbook of Bateries". McGraw-Hill Handbooks, 3 ed ed. (1995).

[19] T. Luo, L. Li, V. Ghorband, Y. Zhan, H. Song \& J.B. Christen. A portable impedance-based electrochemical measurement device. In "2016 IEEE International Symposium on Circuits and Systems (ISCAS)” (2016), pp. 2891-2894. doi:10.1109/ISCAS.2016.7539197.

[20] A. Malik, Z. Zhang \& R.K. Agarwal. Extraction of battery parameters using a multi-objective genetic algorithm with a non-linear circuit model. Journal of Power Sources, 259 (2014), 76-86.

[21] S. Manzetti \& F. Mariasiu. Electric vehicle battery technologies: From present state to future systems. Renewable and Sustainable Energy Reviews, 51 (2015), 1004 - 1012.

[22] W.H. Meyer. Polymer Electrolytes for Lithium-Ion Batteries. Advanced Materials, 10 (1998), 439448.

[23] S. Mousavi \& M. Nikdel. Various battery models for various simulation studies and applications. Renewable and Sustainable Energy Reviews, 32 (2014), 477 - 485.

[24] D. Panigrahi T, D. Panigrahi, C. Chiasserini, S. Dey, R. Rao, A. Raghunathan \& K. Lahiri. Battery life estimation of mobile embedded systems. In "VLSI Design, 2001. Fourteenth International Conference on” (2001), pp. 57-63. doi:10.1109/ICVD.2001.902640.

[25] B.R. Pattipati, C. Sankavaram \& K.R. Pattipati. System Identification and Estimation Framework for Pivotal Automotive Battery Management System Characteristics. IEEE Trans. Systems, Man, and Cybernetics, Part C, 41(6) (2011), 869-884. doi:10.1109/TSMCC.2010.2089979.

[26] D. Rakhmatov \& S. Vrudhula. An analytical high-level battery model for use in energy management of portable electronic systems. National Science Foundation's State/Industry/University Cooperative Research Centers (NSFS/IUCRC) Center for Low Power Electronics (CLPE), (2001), 1-6.

[27] J.E.B. Randles. Kinetics of rapid electrode reactions. Discuss. Faraday Soc., 1 (1947), 11-19.

[28] S.M. Rezvanizaniani, Z. Liu, Y. Chen \& J. Lee. Review and recent advances in battery health monitoring and prognostics technologies for electric vehicle (EV) safety and mobility. Journal of Power Sources, 256 (2014), 110 - 124. 
[29] L.C. Romio, A.T.Z.R. Sausen, P.S. Sausen \& M. Reimbold. "Mathematical Modeling of the Lithiumion battery Lifetime using System Identification Theory". Advances in Mathematics Research. Nova Science Publishers Incorporated (2015), 360-375 pp.

[30] U. Sahapatsombut, H. Cheng \& K. Scott. Modelling of electrolyte degradation and cycling behaviour in a lithium-air battery. Journal of Power Sources, 243 (2013), 409 - 418. doi:https://doi.org/10.1016/ j.jpowsour.2013.06.043.

[31] V. Sangwan, A. Sharma, R. Kumar \& A.K. Rathore. Estimation of battery parameters of the equivalent circuit models using meta-heuristic techniques. In "1st International Conference on Power Electronics, Intelligent Control and Energy Systems (ICPEICES), IEEE” (2016), pp. 1-6. doi:10.1109/ICPEICES. 2016.7853240 .

[32] B. Schweighofer, K.M. Raab \& G. Brasseur. Modeling of high power automotive batteries by the use of an automated test system. IEEE Transactions on Instrumentation and Measurement, 52(4) (2003), 1087-1091. doi:10.1109/TIM.2003.814827.

[33] A. Seaman, T.S. Dao \& J. McPhee. A survey of mathematics-based equivalent-circuit and electrochemical battery models for hybrid and electric vehicle simulation. Journal of Power Sources, 256 (2014), 410 - 423. doi:http://dx.doi.org/10.1016/j.jpowsour.2014.01.057.

[34] B. Suthar, V. Ramadesigan, P.W.C. Northrop, R.B. Gopaluni, S. Santhanagopalan, R.D. Braatz \& V.R. Subramanian. Optimal control and state estimation of lithium-ion batteries using reformulated models. In “American Control Conference, ACC 2013, Washington, DC, USA, June 17-19, 2013" (2013), pp. $5350-5355$.

[35] K. Thirugnanam, J. Ezhil Reena, M. Singh \& P. Kumar. Mathematical Modeling of Li-Ion Battery Using Genetic Algorithm Approach for V2G Applications. Energy Conversion, IEEE Transactions on, 29(2) (2014), 332-343.

[36] O. Tremblay, L.A. Dessaint \& A.I. Dekkiche. A Generic Battery Model for the Dynamic Simulation of Hybrid Electric Vehicles. In "Vehicle Power and Propulsion Conference, 2007. VPPC 2007. IEEE" (2007), pp. 284-289.

[37] C. Zhang, K. Li, S. Mcloone \& Z. Yang. Battery modelling methods for electric vehicles - A review. In “2014 European Control Conference (ECC)” (2014), pp. 2673-2678. doi:10.1109/ECC.2014. 6862541. 